\title{
Influence of Climatic Changes on the Joint Strength of Laser Joined Plastic-Metal-Hybrids
}

\author{
Kira van der Straeten, Alexander Olowinsky and Arnold Gillner \\ Fraunhofer Institute for Laser Technology ILT, Steinbachstr. 15, 52074 Aachen, Germany \\ E-Mail: kira.van.der.straeten@ilt.fraunhofer.de
}

\begin{abstract}
Multi-material lightweight construction enables reducing dead weight while maintaining and preferably boosting the components' performance. The combination of different materials such as fiber-reinforced plastics (FRP) and metals require reliable joining techniques. A promising approach for joining is a two-staged laser-based joining process, which consists of microstructuring the metal surface, followed by thermal joining. In the first step, spongy microstructures with nanosubstructures are generated on the metal surface. In the subsequent joining process polymer and metal are joined via thermal direct joining. Therefor both joining partners are clamped together, the metal surface is heated up with a diode laser and through heat conduction the thermoplastic polymer matrix melts and flows into the structures to harden there. For automotive and aeronautical applications, the faultless use of the connection must also be ensured at varying temperatures. However, plastics and metals have very different coefficients of thermal expansion. In this contribution the influence of climate changes on laser-based FRP-metal hybrid joints is investigated and evaluated. Therefor tensile shear samples, made of stainless steel and glass fiber-reinforced Polypropylene, are stored in a climate chamber for temperature cycles between $-40^{\circ} \mathrm{C}$ up to $80^{\circ} \mathrm{C}$. The joint strength is tested before and after climate change and the joining interface is analyzed with cross-sections.
\end{abstract}

DOI: 10.2961/jlmn.2018.03.0024

Keywords: lightweight, hybrid joining, laser joining, plastic-metal-joining, surface texturing, conelike protrusions, climatic changes

\section{Introduction}

Lightweight construction is a central issue in automotive and aerospace industry due to the need to reduce $\mathrm{CO}_{2}$ emissions. One way to achieve weight reduction is to use different materials adapted to functions and local loads. The multi-material lightweight approach enables reducing dead weight while maintaining and preferably boosting the components' performance. However, the combination of different materials such as fiber-reinforced plastics (FRP) and metals require reliable joining techniques, because a direct connection fails due to their different chemical and physical properties. While adhesive joining typically requires long process times caused by extensive surface pretreatment and hardening times, fastening and riveting is not suitable for fiber-reinforced plastics due to the punctual load transfer. A promising alternative is a novel, two-staged laser-based joining process, which consists of microstructuring the metal surface, followed by thermal joining. In the first step, spongy, randomly orientated microstructures with nano-substructures are generated on the metal surface. During ablation of metals with ultrashort pulsed laser radiation self-organizing microstructures appear, which are called cone-like protrusions (CLP) [1]. These microstructures enlarge the boundary surface and create interlocking points for the polymer. In the subsequent joining process polymer and metal are joined via thermal direct joining. Therefor both joining partners are clamped together, the metal surface is heated up with a diode laser and through heat conduction the thermoplastic polymer matrix melts and flows into the structures. After hardening, a strong and durable connection between both materials is formed which is mostly based on interlocking between the materials. With this joining technique tensile shear strengths above $25 \mathrm{MPa}$ have been demonstrated [2].

\section{Fundamentals}

The laser based process chain for joining polymers with metals is shown in Fig. 1. During the first process step the metal surface is microstructured with laser radiation to enlarge the active surface and create undercut structures for interlocking the polymer into the metal. In a second heat conduction joining step the metal is heated up e.g. using laser radiation. Through the thermal contact of polymer and metal the thermoplastic composite material melts. An external joining force is applied and the molten material flows into the structures. After hardening a durable and strong connection is formed.

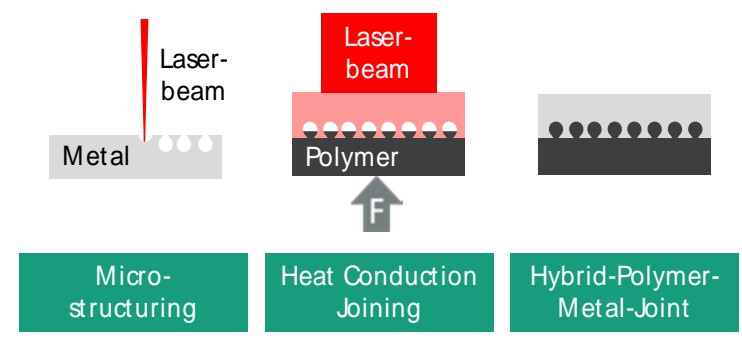

Fig. 1 Process chain for laser-based plastic-metal-joining. 
For the metal surface pretreatment, different lasermicrostructuring approaches are investigated, e.g. the formation of undercut grooves with cw-laser radiation [3]. In this contribution ultrashort laser pulses are used to create a spongy surface with so-called cone-like protrusions (CLP, Fig. 2). During the ablation of metals or silicon with ultrashort laser pulses the formation of numerous self-organized microstructures can be observed e.g. mounds, spikes, micro- and nanoripples or CLP $[4,5]$.
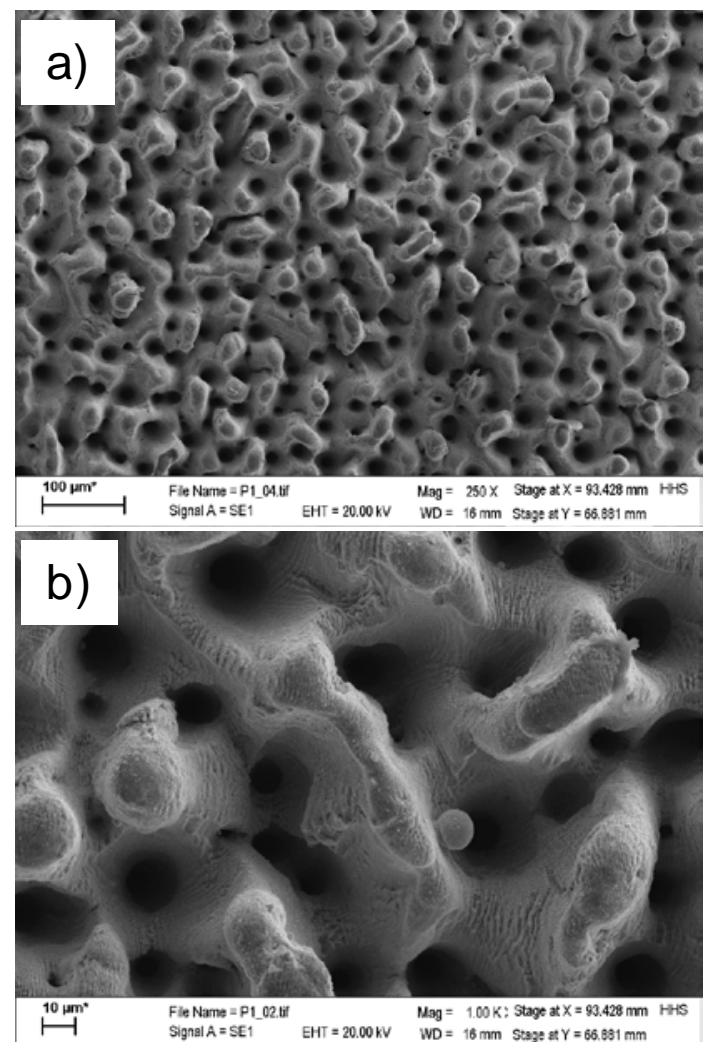

Fig. 2 Cone-like Protrusions (CLP) on steel (SEM picture) at 250 (a) and 1000x (b) magnification.

The formation of CLP can be observed at medium $\left(\sim 1 \mathrm{~J} / \mathrm{cm}^{2}\right)$ and high fluences on steel, aluminum, titanium and silicon [1, 6]. Usually the effect starts at grooves, scratches and inhomogeneities after a few layers of ablation with high energy ultrashort pulses in the ablated area. At first some dots and holes appear which grow to small clusters with increasing number of layers. Visually the growth of the CLP can be monitored due to a blackening of the surface. The structure is characterized by a random orientation and an extremely enlarged surface with a high surface roughness.

\section{Experimental Setup and Procedure \\ 3.1 Materials and Sample Geometry}

The metal used for the hybrid joint is a commercially available stainless steel X5CrNi18-10 (1.4301). During the joining process the stainless steel is combined with a glass fiber reinforced Polypropylene (PP) for tensile shear tests which are also used for climate change tests.

The glass fiber reinforced plastic (GFRP) used is X111F40-4/1-0/90 (Quadrant Plastic Composites AG, Switzerland), which is a chopped fiber glass mat reinforced
PP laminate with randomly oriented glass fibers and additionally reinforced fabric inside. The material thickness is 3 $\mathrm{mm}$ with a glass fiber content of 40 vol.-\% (PP/GF40).

In this contribution the sample geometry of overlap tensile shear samples is used to evaluate the performance of the joint before and after climate change tests. The dimensions and the sample arrangement of the joining partners are depicted in Fig. 3. The dimensions of the samples are changed from standard $25 \times 12.5 \mathrm{~mm}^{2}$ overlap to a reduced overlap of $5 \mathrm{~mm}$. This is necessary to avoid the failure of the basic material outside the joint during testing. The width is increased to $30 \mathrm{~mm}$.

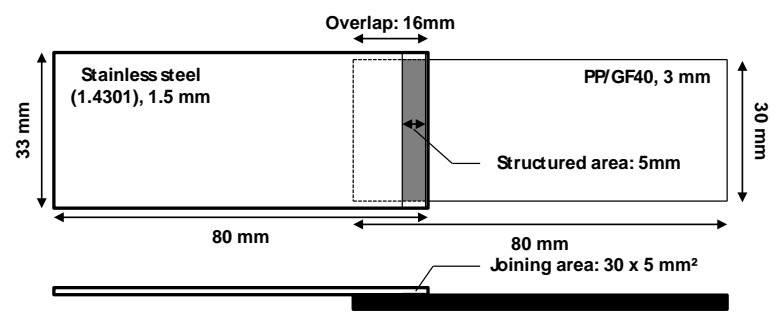

Fig. 3 Sample geometry and arrangement.

\subsection{Experimental Setup for Microstructuring and Joining}

The laser microstructuring process of the metal surface is conducted with a laser system consisting of a Kugler Microgantry nano3X (3-axis motion system) with an ultrashort pulsed laser Hyper Rapid 100 (Coherent). Pulse duration is $<15 \mathrm{ps}$ at a wavelength of $\lambda=1064 \mathrm{~nm}$. The maximum average output power is $100 \mathrm{~W}$ at a repetition rate of $400 \mathrm{kHz}$. After deflection by a galvanometric scanner (IntelliScan14, Scanlab), the laser beam is focused through a lens ( $\mathrm{f}=163 \mathrm{~mm}$ ) on the work piece to a spot size of $\square$ pprox.. $34 \mu \mathrm{m}$. Parameters that can be varied to optimize the process window for the formation of CLP are scanning speed v, structure offset SO, laser power $\mathrm{P}$ and the number of ablated layers $\mathrm{N}$. The samples are processed with a crossed scanning hatch (see Fig. 3).
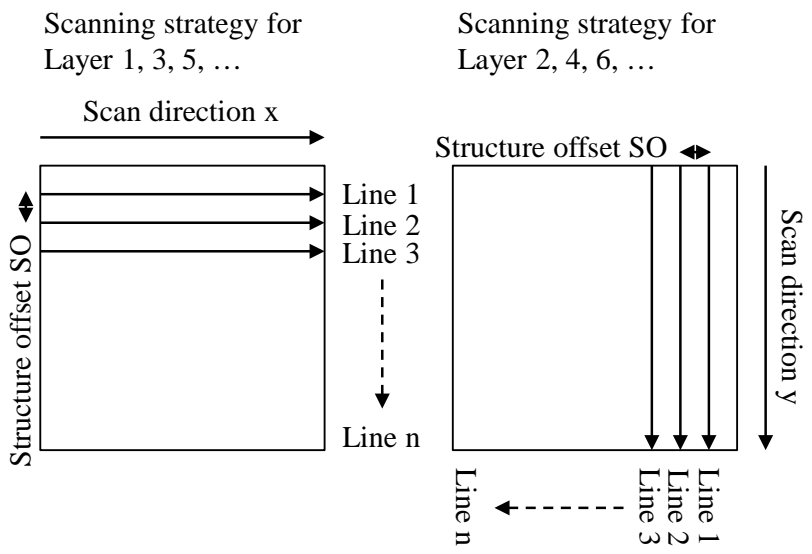

Fig. 4 Scanning strategy for microstructuring.

The choice of microstructuring parameters are based on preliminary tests. The parameters for microstructuring are listed in Table 1. These parameters have proven to provide a full coverage of the surface with CLP structures as seen in Fig. 2. 
Table 1 Laser microstructuring parameters.

\begin{tabular}{|c|c|c|c|}
\hline $\begin{array}{c}\text { Av. Laser } \\
\text { Power } \\
{[\mathrm{W}]}\end{array}$ & $\begin{array}{c}\text { Scanning } \\
\text { speed } \\
{[\mathrm{mm} / \mathrm{s}]}\end{array}$ & $\begin{array}{c}\text { Structure } \\
\text { offset } \\
{[\mu \mathrm{m}]}\end{array}$ & $\begin{array}{c}\text { Ablated } \\
\text { layers } \\
{[\#]}\end{array}$ \\
\hline 57 & 2000 & 8 & 38 \\
\hline
\end{tabular}

The laser system used for the joining process is a Laserline GmbH LDM 3000-100 continuous wave diode laser combined with a MOTOMAN-HP20-6 robot motion system (Fig. 5). The maximum output power is $\sim 3000 \mathrm{~W}$ and the laser system is operating at $\lambda=900-1070 \mathrm{~nm}$ wavelength. A zoom optics from Laserline with a focal length of $250 \mathrm{~mm}$ forms a rectangular laser spot with a variable size from $5 \times 5 \mathrm{~mm}^{2}$ up to $16 \times 30 \mathrm{~mm}^{2}$. The variable spot size enables simultaneous irradiation of the entire joining area with a single pulse adapted to the sample geometry. In order to apply pressure and fix the sample arrangement, a pneumatic clamping device with a sample holding fixture is used. A clamping pressure of 3 bars with a pneumatic cylinder of $50 \mathrm{~mm}$ diameter is applied on the joining area. While the joining pressure is applied, the joining zone is simultaneously irradiated on the metal side. Through heat conduction, the plastic melts and flows into the structures.

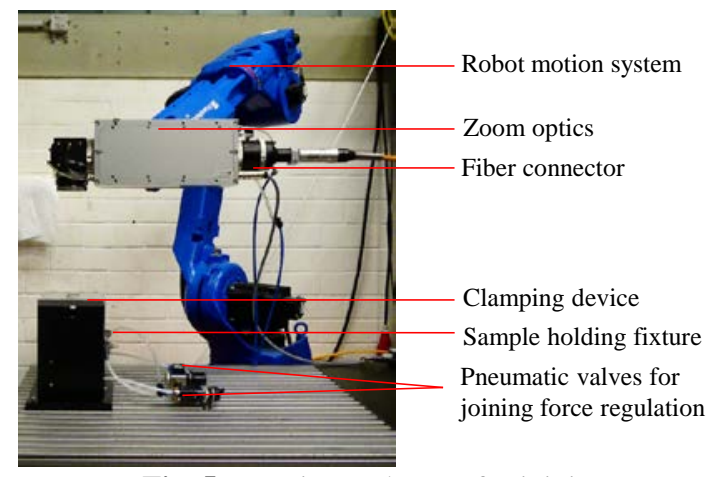

Fig. 5 Experimental setup for joining.

The parameters chosen for joining are based on preliminary tests and are listed in Table 2. The parameters enable melting the polymer in the complete joining area without a decomposition of the matrix. These parameters are used to fabricate 36 samples for further testing (6 sets of 6 samples: 5 for tensile shear strength testing and 1 for cross-sections).

Table 2 Laser joining parameters.

\begin{tabular}{|c|c|c|}
\hline $\begin{array}{c}\text { Laser Power } \\
{[\mathrm{W}]}\end{array}$ & $\begin{array}{c}\text { Irradiation Time } \\
{[\mathrm{ms}]}\end{array}$ & $\begin{array}{c}\text { Joining Area } \\
{\left[\mathrm{mm}^{2}\right]}\end{array}$ \\
\hline 3000 & 2300 & $5 \times 30=150$ \\
\hline
\end{tabular}

\subsection{Climate Change Tests}

The climate change tests were conducted using a climatic chamber ClimeEvent C/180/40/3 (Weiss Umwelttechnik, Fig. 6). This climatic chamber operates at temperatures between $-42^{\circ} \mathrm{C}$ and $180^{\circ} \mathrm{C}$ and $10-98 \%$ rel. humidity. The maximum average heating and cooling rate is $4 \mathrm{~K} / \mathrm{min}$.

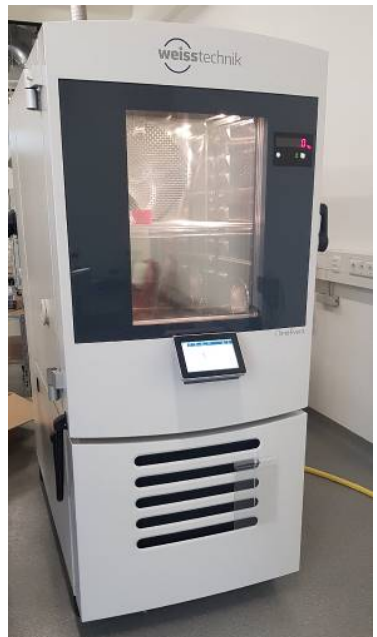

Fig. 6 Climatic chamber (Weiss Umwelttechnik).

In this contribution a cycle of 4 hours with temperatures between $-40^{\circ} \mathrm{C}$ and $80^{\circ} \mathrm{C}$ and min. humidity $(0 \%)$ is set up, which consists of 4 steps listed in Table 3 . The temperature of $80^{\circ} \mathrm{C}$ is chosen based on the maximum operating temperature for PP. The tests are not defined for a specific application and are therefore not carried out according to a specific directive.

Table 3 Process steps for one climate changing cycle.

\begin{tabular}{|l|l|}
\hline Step & Parameters \\
\hline 1 & Heating up to $80^{\circ} \mathrm{C}$ in $1 \mathrm{~h}$ at min. humidity \\
\hline 2 & Holding $80^{\circ} \mathrm{C}$ for $1 \mathrm{~h}$ at min. humidity \\
\hline 3 & Cooling down to $-40^{\circ} \mathrm{C}$ in $1 \mathrm{~h}$ at min. humidity \\
\hline 4 & Holding $-40^{\circ} \mathrm{C}$ for $1 \mathrm{~h}$ at min. humidity \\
\hline
\end{tabular}

This cycle is carried out 6 times a day (24h) for up to 15 days. The 30 samples (5 sets) are placed inside the climatic chamber during the testing period, the last set is used for comparison and reference. Sample sets are taken and tested at different points in the test cycle and compared to the reference set. Sets are taken out after 1 day ( 6 cycles), 3 days (18 cycles), 5 days (30 cycles), 10 days (60 cycles) and 15 days ( 90 cycles).

The mechanical properties (tensile shear strength) of the hybrid joints are tested with a multi-purpose testing machine Z100 (Zwick GmbH \& Co. KG). For the shear strength tests a tensile force is applied parallel to the joining area and the breaking force is measured. The samples are fixed between the clamping jaws at $50 \mathrm{~mm}$ distance. The testing speed is set to $50 \mathrm{~mm} / \mathrm{min}$. The influence of the climate change test on the hybrid joint interface is evaluated by cross sections and their microscopically analysis.

\section{Results and Discussion}

\subsection{Microstructuring and Joining Results}

During ablation of the steel surface with ultrashort laser pulses, CLP structures grow on the surface as described in previous works $[1,2,7]$. These spongy microstructures (see Fig. 2), which are additionally covered with nanosubstructures, are characterized by a random orientation and a high surface roughness. Due to deep holes, undercuts and the extremely enlarged surface the microstructured surface offers interlocking points and good conditions for joining with thermoplastic polymers. 


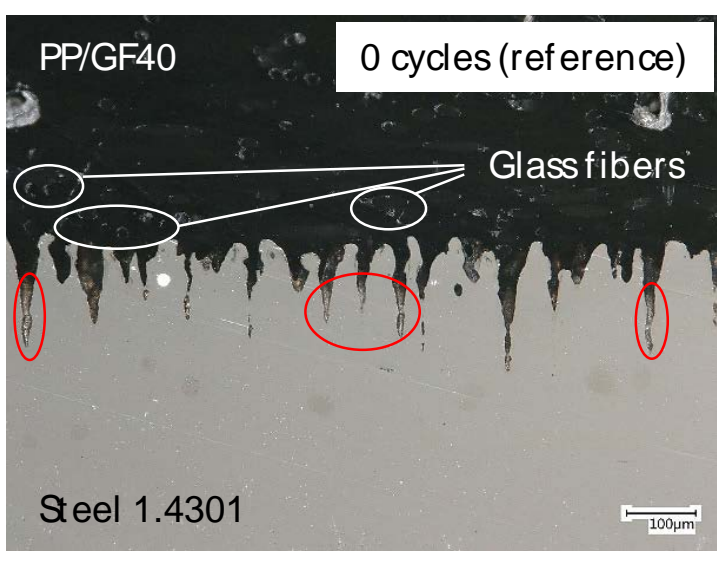

Fig. 7 Cross-section of the reference joint (0 temperature cycles).

In the subsequent joining process the metal is heated up via diode laser radiation and through heat conduction the polymer melts and flows into the structures. In Fig. 7 a cross-section of a hybrid joint between stainless steel 1.4301 and PP/GF40 without thermal cycling tests is shown. The microstructures are up to $180 \mu \mathrm{m}$ deep at a low width between a few microns up to $30 \mu \mathrm{m}$. This results in an aspect ratio (depth to width) $>5$. With the selected laser parameters the cavities are not completely filled with matrix material (red circles, Fig. 7), which is usually caused by insufficient joining pressure or too short irradiation times. The resulting air pockets are usually located in the tips of the structures. The glass fibers, which are the white speckles in the cross-sections, seem not to be moving deep into the cavities. This is best seen in Fig. 8 for 30 cycles.

\subsection{Climate Change Test Results}

Hybrid joints of plastics and metals are used under different environmental conditions, e.g. different temperatures, especially in automotive industry. Both materials have very different coefficients of thermal expansion, which can lead to problems regarding long-term stability of the joints. For laser-based direct joints between plastics and metals no additional material is used, which could buffer the thermal expansion in the joint interface. That is why information about the influence of thermal cycling on the joint strength and quality is essential for further use.

Climate change tests are performed as described in 3.3 with thermal cycles between $-40^{\circ} \mathrm{C}$ and $80^{\circ} \mathrm{C}$. Samples are taken out of the chamber, analyzed and tested after 6,18 , 30, 60 and 90 cycles. The cross-sections of the hybrid joints after thermal cycling are shown in Fig. 8. These cross-sections do not show any changes compared to the reference sample (Fig. 7). The structures are mostly but not completely filled with matrix material, especially in the tips of the structures. Fibers do not move into the cavities. There are no areas visible which are delaminated due to thermal expansion of the different materials.

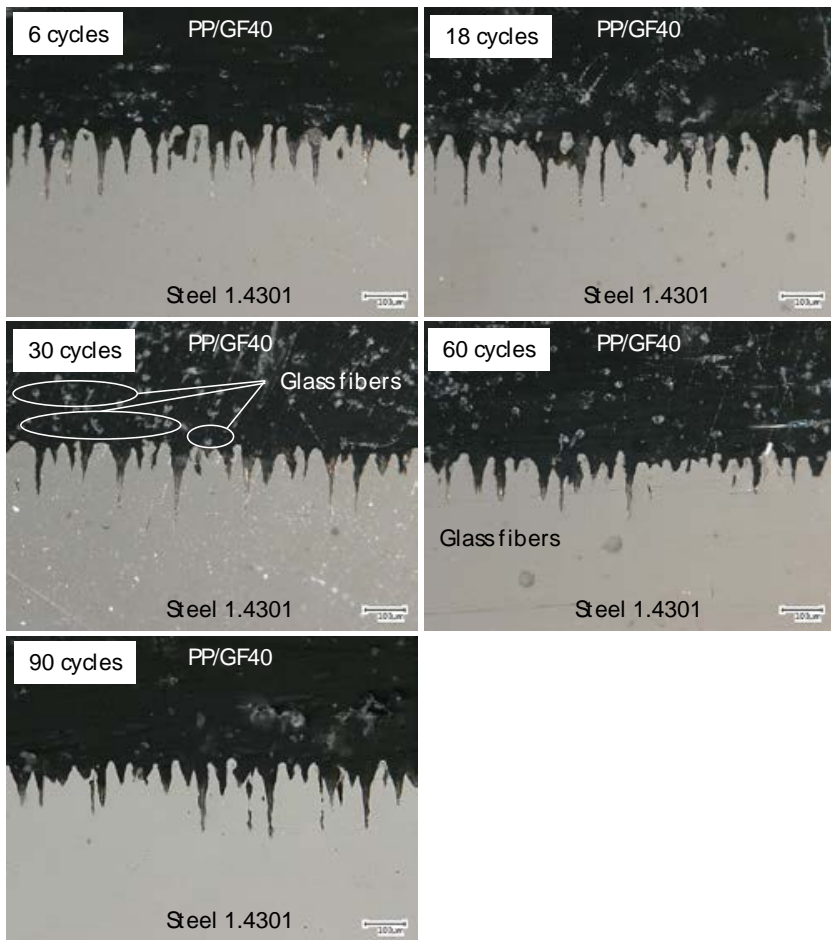

Fig. 8 Cross-sections of hybrid joints after climate changing tests.

After cross-section analysis of the joint, the samples are tested for tensile shear strength as described. The breaking forces are varying between 2500 and $3500 \mathrm{~N}$. The results for tensile shear strength before and after thermal cycling are depicted in Fig. 9.

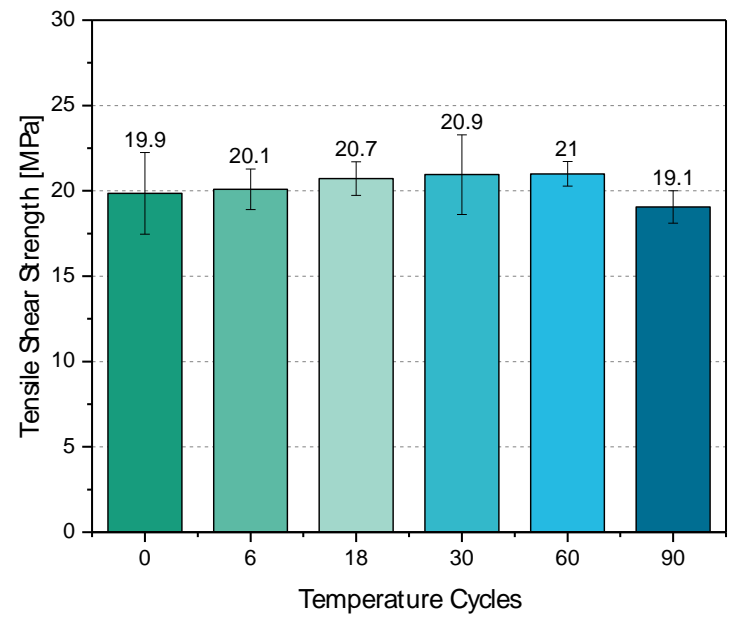

Fig. 9 Tensile shear strength of hybrid joints before and after climate change tests.

The strength of the hybrid joint is approx. $20 \mathrm{MPa}$ before climate changing. Unlike expected due to the difference in thermal expansion of both materials, the tensile shear strength does not decrease after exposure to climate change. With up to $21 \mathrm{MPa}$ the values are even slightly higher except the 90 cycle-samples, but are all in the range of the standard deviation of the tested samples. The difference in joint strength for the longest testing cycle is not sufficient to show a significant decrease in joint strength after climate change tests. This reflects the analysis of the cross-sections which do not show any difference in the joint interface. 
Compared to previous works [7] the average tensile shear strength is slightly lower than expected (20 MPa compared to $24.5 \mathrm{MPa}$ ). This might be caused by the not completely filled cavities, due to insufficient joining pressure or too short irradiation times, although we have still a cohesive failure of the GFRP (see Fig. 10)
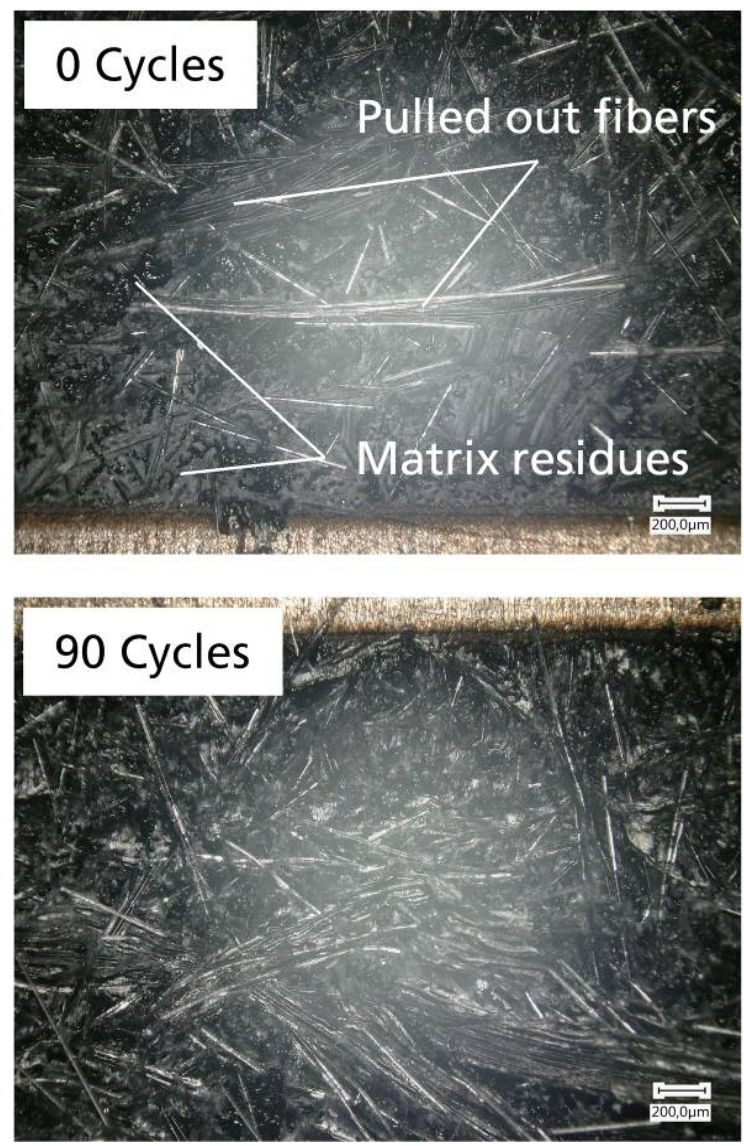

Fig. 10 Fracture area of a hybrid joint after 0 and 90 cycles.

The fracture area of hybrid joint after 0 and 90 temperature cycles is depicted in Fig. 10. The fracture area of the samples shows a cohesive failure of the PP/GF40. Matrix residues are still on the microstructured metal surface and partially even fibers are pulled out of the composite material. The microstructures are completely covered with matrix material. There is no visible difference between the fracture surfaces of thermally loaded and unloaded samples.

In general it can be concluded that climate change has no visible influence on the joint in terms of the contact surface or tensile shear strength of the laser-based hybrid joints between stainless steel and glass fiber reinforced PP. These good results are attributable especially to the high flexibility of the PP but also to the robustness of the stainless steel. They also match other investigations of Aluminum-PP-joints during climate change [8]. However, climate changes might have a greater impact on other material combinations in terms of bond strength and stability.

\section{Summary and Outlook}

Laser-based joining of plastics and metals enables connecting both material without any additional materials such as adhesives or fasteners. In order to achieve a connection, first, the surface of stainless steel (1.4301) is ablated with ultrashort laser pulses to create a spongy surface. This structures consists of so-called Cone-like Protrusions (CLP), self-organizing microstructures which are covered with nano-substructures. These structures extremely enlarge the boundary surface and create interlocking points for the subsequent joining process.

In the second process step plastic and metal are joined together via heat conduction joining using a diode laser source. The molten polymer (PP/GF40) flows into the structures and hardens there, which creates a strong connection between metal and polymer based on mechanical interlocking and specific adhesion.

These joints have already proven to feature very high joint strengths [2, 7]. Due to the different coefficients of thermal expansion of metals and polymers, information about the aging behavior of the joint during climate change are necessary for further use of the technology. In this contribution stainless steel-PP/GF40 tensile shear samples are thermally cycled between $-40^{\circ} \mathrm{C}$ and $80^{\circ} \mathrm{C}$ for up to 90 temperature cycles. The influence on the joint is investigated via cross-sections and testing of the joint strength.

The climate change tests do not show any influence on neither the joint interface nor the tensile shear strength. The joint strength before and after climate change is approx. $20 \mathrm{MPa}$. These results are very promising for using laserbased joining techniques for industrial applications such as in automotive industry. Still, other material combinations which might be more affected from climate change, e.g. due to aging of the thermoplastic material or delamination in the joining area should be investigated. Additionally the other aging factors like corrosion are a big issue for some material combinations, which should be pursued to enable a safe use in the industry.

\section{Acknowledgments}

Our thanks go to Fabian Platzbecker who performed most of the experiments.

\section{References}

[1] M. Tsukamoto, T. Kayahara, H. Nakano, M. Hashida, M. Katto, M. Fujita, M. Tanaka and N. Abe. J. Phys., Conference Series 59, (2007) 666

[2] K. van der Straeten, I. Burkhardt, A. Olowinsky and A. Gillner. Phys. Procedia, 83, (2016) 1137

[3] C. Engelmann, J. Eckstaedt, A. Olowinsky, M. Aden and V. Mamuschkin. Phys. Procedia, 83, (2016) 1118

[4] C.A. Zuhlke, T.P. Anderson and D.R. Alexander. Opt. Express, 21, (2013) 8460

[5] B. Raillard, L. Gouton, E. Ramos-Moore, S. Grandthyll, F. Müller and F. Mücklich. Surf. Coat. Technol., 207, (2012) 102

[6] S. Eifel, A. Dohrn and A. Gillner. Proceedings of LPM2010 (2010), Stuttgart

[7] K. van der Straeten, A. Olowinsky and A. Gillner., J. Laser Appl., 30, No.3, (2018) 032401-1

[8] A. Heckert, C. Singer, M. F. Zaeh, R. Daub and T. Zeilinger. Phys. Procedia, 83, (2016) 1083 